\title{
Application of Data Fusion Technology in Greenhouse Environment Monitoring and Control System
}

\author{
Xiangfei Meng ${ }^{1,2}$ and Changming Wang ${ }^{1}$ \\ ${ }^{1}$ School of Mechanical Engineering, NUST, Nanjing 210094, China \\ ${ }^{2}$ Changshu Institute of Technology, Changshu, 215500, China
}

\begin{abstract}
Multisensor data fusion technology is a new frontier technology. According to the demand of greenhouse environment monitoring, the paper puts forward a new kind of data fusion method based on Dempster-Shafer (D-S) evidence theory and agricultural expert system. The experimental results show that the method can improve the veracity of decision of measuring and controlling greenhouse environment parameters, and can significantly improve the control effect of greenhouse environment.
\end{abstract}

Keywords: greenhouse environment, multisensor data fusion, D-S evidence theory, agricultural expert system.

\section{Introduction}

The environmental conditions in the greenhouse play an important role in crop growth and development. It is a key point of the development of greenhouse technology that how to make the most suitable greenhouse environment for crop growth. Now a single sensor is mainly used to monitor the greenhouse environment, such as temperature monitoring, humidity monitoring, $\mathrm{CO}_{2}$ monitoring, soil moisture monitoring, illumination monitoring, etc, since the lack of collaboration processing and comprehensive utilizing the multisource and multidimensional information, there are varied defects in accuracy, reliability, practicability, etc. By better utilizing the information resources, data fusion technology is applied to the greenhouse environment monitoring and control system to incorporate and corresponding process multidimensional information from various sensors [1]. So more accurately reflection about the greenhouse environment condition can be got and more accurately and reliably greenhouse environment monitor can be carried out.

\section{Data Fusion Technology}

\subsection{Data Fusion Process}

Data fusion is the technology which makes full use of multisensor resources. It can combine the information, which is redundant and complementary in time and space, according to certain standards, by using the multisensor and observation information properly to get the consistent explanation or description of the tested object. The 
processes of Data fusion mainly includes multisensor (signal acquisition), data preprocessing, data fusion center (feature extraction, data fusion Calculation) and result output [2].

\subsection{Fusion Algorithm}

As a data processing technology, data fusion is in fact the integration and application of traditional science and new technologies. Traditional multiple sensor fusion mainly includes simple filtering method, the weighted averages method, production rules, the Bayesian estimation, D-S evidence reasoning method, etc. In recent years, many new fusion methods have also been put forward, including expert system, neural network and fuzzy theory, etc. This paper put forward a kind of fusion method based on the evidence theory and the expert system to monitor the greenhouse environment [3].

The biggest characteristic of D-S evidence theory structure is introducing the uncertainty into evidence and establishing the axioms. For example, basic probability assignment function $(B P A F)$, believe function $(B E L)$, plausibility function $(P L)$, etc. The most basic concept of D-S evidence theory is the recognition framework, recorded as $\Theta . \Theta$ represents the set of all possible proposition in a domain, Every proposition is represented as a subset of $\Theta .2^{\Theta}$ is $\Theta$ Power set and makes up a set of proposition $\Omega(\Theta)$. If the elements meet the condition that incompatible with each other in a discernment frame, the $m(A)$, values $A$ assigns to function $m$, is a mapping form power set to [0,1]. If the function $m$ meets the following conditions:

$$
\begin{gathered}
m(\Phi)=0 \\
\forall A \in 2^{\Theta}, m(A) \geq 0, \text { and } \sum_{A \in 2^{\Theta}} m(A)=1
\end{gathered}
$$

$m(A)$ can be called as a basic probability assignment function of $A$. Among them $\Phi$ represents empty set, $m(A)$ represents the precise degree of believe in proposition $A$ and the directly support of $A$.

For a given basic probability assignment function $m$ and $\forall A \in 2^{\Omega}$, the corresponding belief function is defined as:

$$
\operatorname{Bel}(A)=\sum_{B \subseteq A} m(B)
$$

Plausibility function is defined as:

$$
P l(A)=1-\operatorname{Bel}(\bar{A})=\sum_{B \cap A \neq \Phi} m(B)
$$

$\mathrm{Bel}$ function is called as lower limit function, expressed the degree of believe in proposition $A$, including $\operatorname{Bel}(\Phi)=0, \operatorname{Bel}(\Omega)=1 . P l$ function also known as the upper limit function or not negative function, it is a sum of all the set of basic probability assignment functions that meet $A$. As the evidence refused to $A, P l(A)=0$, when there is no evidence against $A, P l(A)=1$, it is easy to prove $P l(A) \geq \operatorname{Bel}(A)$. 
[Bel (A), $P l(A)]$ represents the belief range of $A$. If $A$ is a subset in discernment frame and $m(A)>0, A$ is called as a focal of belief function $\mathrm{Bel}$. In this way, trust degree and plausibility have summarized the relations evidence of proposition $A$, the relations between them as shown in figure 1; this constitutes a complete range of evidence.

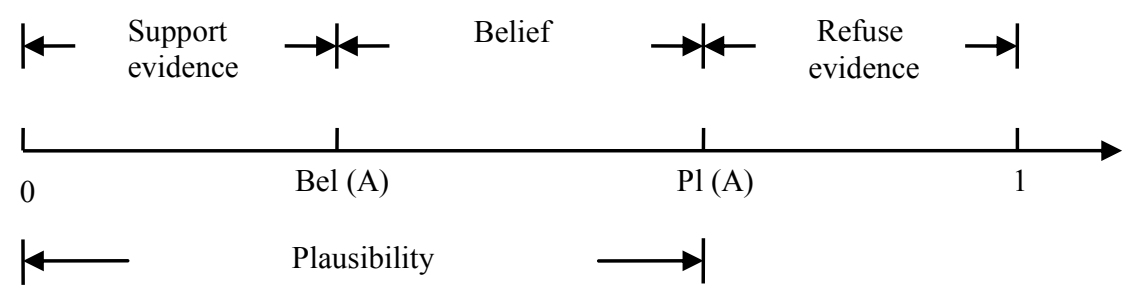

Fig. 1. Evidence range schemes

Basic rules of D-S evidence combination: Hypothesizing that there are two reasoning system, their basic probability assignment and belief function is $m_{1}, m_{2}$ and $\mathrm{Bel}_{1}, \mathrm{Bel}_{2}$ respectively, for subset $A$, the D-S rules composed of probability assignment of the two reasoning system:

$$
m(A)=\frac{\sum_{A_{1} \cap A_{2}=A} m_{1}\left(A_{1}\right) m_{2}\left(A_{2}\right)}{\sum_{A_{1} \cap A_{2} \neq \Phi} m_{1}\left(A_{1}\right) m_{2}\left(A_{2}\right)}=m_{1}\left(A_{1}\right) \oplus m_{2}\left(A_{2}\right)
$$

The corresponding $\mathrm{Bel}$ of $m$ called synthesis or sum value of $\mathrm{Bel}_{1}$ and $\mathrm{Bel}_{2}$, recorded as $\mathrm{Bel}=\mathrm{Bel}_{1} \oplus \mathrm{Bel}_{2}$.

$$
\sum_{A_{1} \cap A_{2} \neq \Phi} m_{1}\left(A_{1}\right) m_{2}\left(A_{2}\right)=1-\sum_{A_{1} \cap A_{2}=\Phi} m_{1}\left(A_{1}\right) m_{2}\left(A_{2}\right)=1-k
$$

$1-k$ is a correction factor. In fact, the introduction of $1-k$ is in order to avoid assigning nonzero probabilities to empty sets when combining the evidences, so that the abandoned belief assignment of empty sets can be added to nonvoid set proportionally. The $k$ objectively reflects the degree of conflict among evidences during the date fusion process. The range of $k$ is $0 \leq k \leq 1$, the bigger $k$ is the greater conflict will be. If $k$ close to 1 , it will probably produces unreasonable results and leads to the fusion decisions against intuition; If $k=1, \mathrm{D}-\mathrm{S}$ theory cannot be use to fuse.

\subsection{Expert System}

Expert system is a branch of artificial intelligence. Its main purpose is to make the computers play the role of human experts in each field. It is a kind of intelligence program subsystems, which has a large number of domain knowledge and experiences on expert level. The expert system can solve the problems in the field by using the knowledge and methods that human experts will adopted. It is a computer 
program, which can finish general or humanoid simulation problem-solving strategies on expert level and combine the problem with operational knowledge and experience knowledge [4].

Expert system generally consists of knowledge base, database, inference engine, explain part and the part of the knowledge acquisition. Its core is the knowledge base and Inference engine. The ways that expert system works can be simply boiled down to two parts: knowledge using and reasoning. The basic framework as shown in figure 2:

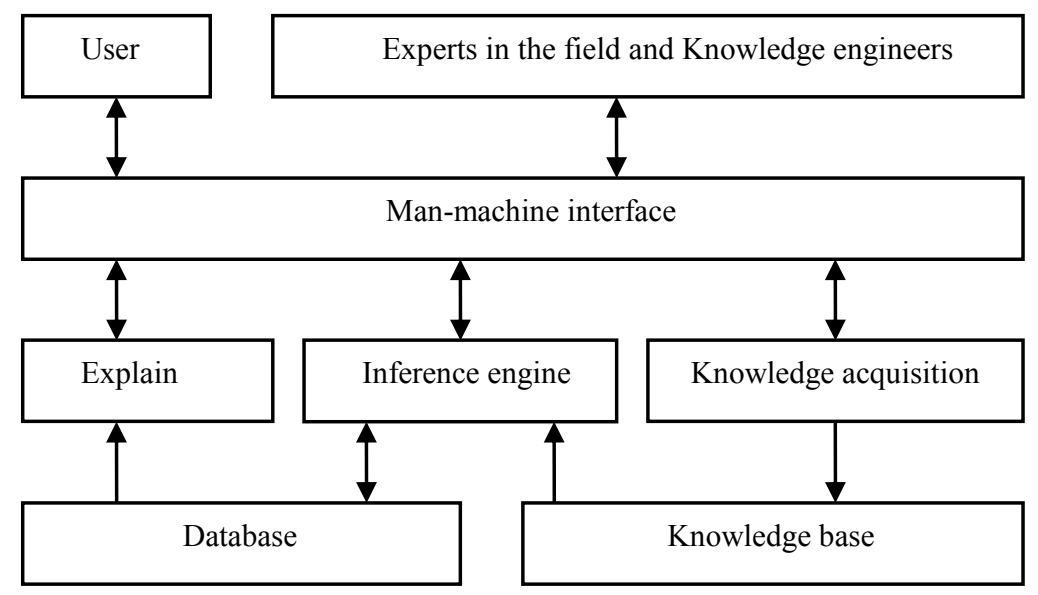

Fig. 2. The basic framework of expert system

\section{The Greenhouse Environment Monitoring System Based on Multisensor Data Fusion}

Environmental monitoring technology is the core of greenhouse technology. In a modern large greenhouse, all the environmental factors such as indoor temperature, light, wet and heat detecting, sensing and adjusting are managed by the computer comprehensively and controlled automatically [5]. The purpose of greenhouse environment controlling is overcome some or entire climate environment in outside world and the objective restrictions, according to the physiological property of different crop, to build a best environment for different crop to growth. At present, the greenhouse environment controlling mostly uses industrial control system. On the one hand, the industrial control system costs more, can't meet the low-cost requirement of establishment agriculture; on the other hand, it focuses on the computer technology during the development process, without the participation of agricultural expert system. It also lacks the expert system that includes physiological information of crops. Because of these reasons, the greenhouse environment controlling has some limitations in the management application [6] [7].

This paper adopts a method, which combined the D-S evidence theory with expert system, to do the fusion processing on the three main environment parameter, temperature, humidity and light intensity in greenhouse. According to what may happen 
in the greenhouse environment, a definition of the rational recognition framework can be worked out form expert knowledge, and the assignment on basic probability assignment can be down on the date which is fused locally. And then initial conditions of D-S theory can be acquired. After D-S evidence theory combination rules fusion, all the fusion results will be sent into the knowledge base of expert system to combine with the experiences of domain experts as the basis of inference in the expert system so that a accurate judgment will be worked out, and appropriate measures will be applied to adjust environmental parameters. Finally, the best environment conditions for crop to growth will be acquired. System structure fusion as shown in figure 3:

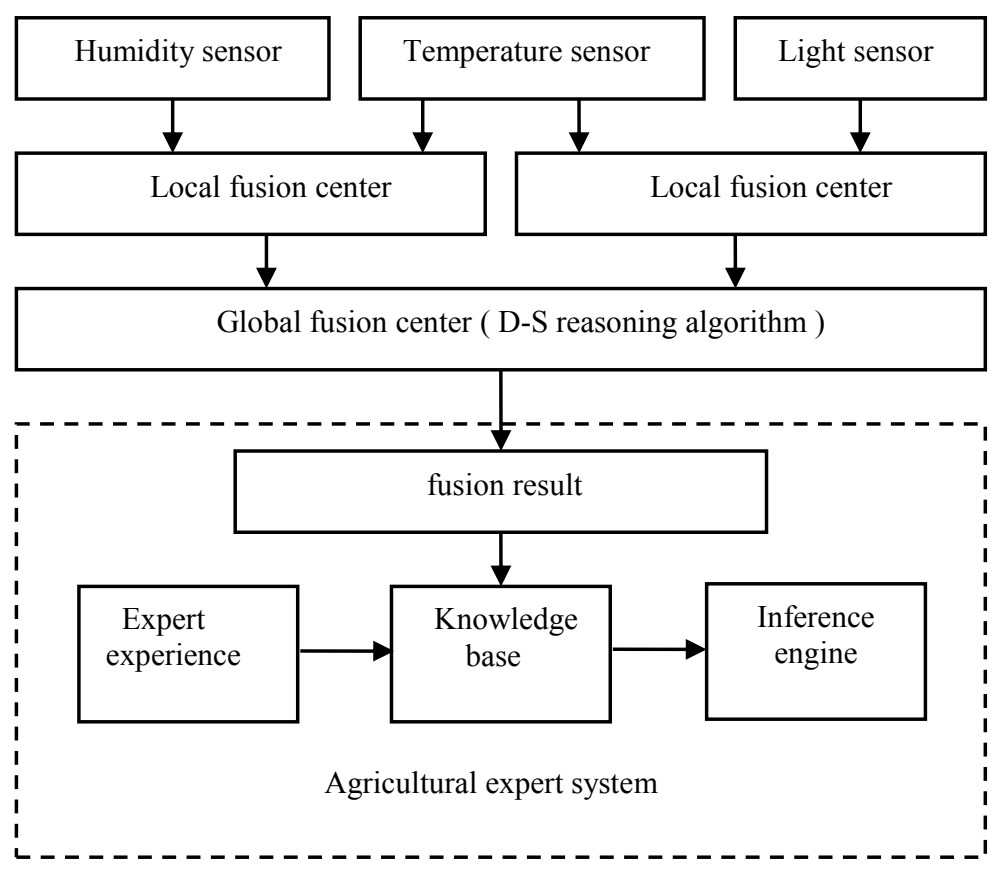

Fig. 3. Structure diagram of system fusion

\section{The Experiment and Application Study for Greenhouse Cultivation}

The simulation experiment of fusion algorithm has been down on specific environmental conditions of strawberry cultivation in a greenhouse trellis. Strawberry belongs to the thermophilic plants. A group of environmental data of greenhouse strawberry cultivation in March is collected for experiment. There is only one or two of the environmental factors play a leading role under certain conditions in the greenhouse. We call them the leading factor. During fruiting period of strawberry, temperature is the leading factor, which affects the yield and quality of strawberry, in sunlight greenhouse. So recognition framework can be built with the knowledge and experiences of domain expert. The recognition framework is as follows: 
$\Theta=\{A, B, C, D, E\}$

$A=\left\{\right.$ appropriate, temperature $20^{\circ} \mathrm{C} \sim 25^{\circ} \mathrm{C}$, humidity $60 \% \sim 70 \%$, light intensity 2500 $\sim 3000 l x\}$;

$B=\{$ low temperature $\}$;

$C=\{$ low temperature, humidity is on the high side $\}$;

$D=\{$ low temperature, light intensity is insufficient $\}$;

$E=\{$ low temperature, humidity is on the high side, light intensity is insufficient $\}$;

Take a group of greenhouse parameters got form 16:30 to 18:30 in the afternoon $\left\{\right.$ temperature $18^{\circ} \mathrm{C}$, humidity $65 \%$, light intensity $\left.1500 l x\right\}$ to do the evidence theory fusion experiments. The fusion results as shown in table 1 and table 2 :

Table 1. Temperature and Humidity fusion

\begin{tabular}{ccccccc}
\hline $\boldsymbol{k}=0.1812$ & $\boldsymbol{A}$ & $\boldsymbol{B}$ & $\boldsymbol{C}$ & $\boldsymbol{D}$ & $\boldsymbol{E}$ & $m(\Theta)$ \\
\hline Temperature & 0.645 & 0.103 & 0.025 & 0.148 & 0.075 & 0.018 \\
Humidity & 0.382 & 0.067 & 0.460 & 0.042 & 0.115 & 0.027 \\
Fusion result & 0.844 & 0.018 & 0.116 & 0.016 & 0.015 & 0.009 \\
\hline
\end{tabular}

Table 2. Temperature and Light intensity fusion

\begin{tabular}{rcccccc}
\hline $\boldsymbol{k}=0.1125$ & $\boldsymbol{A}$ & $\boldsymbol{B}$ & $\boldsymbol{C}$ & $\boldsymbol{D}$ & $\boldsymbol{E}$ & $m(\Theta)$ \\
\hline Temperature & 0.645 & 0.103 & 0.025 & 0.148 & 0.075 & 0.018 \\
Light intensity & 0.053 & 0.104 & 0.019 & 0.702 & 0.058 & 0.001 \\
Fusion result & 0.126 & 0.056 & 0.002 & 0.743 & 0.002 & 0.006 \\
\hline
\end{tabular}

It can be inferred from the above two tables that $m(\Theta)$ was decreased. That means data fusion reduce the uncertainty of the system. At the same time, the fusion of the basic possibility is better separability than before fusion. Table 1 shows that after merging the temperature and humidity, basic probability function value of $A$ is the largest one, and is more than before fusion temperature and humidity their basic possibility function value is bigger. Table 2 shows that after merging the light intensity and temperature, basic possibility function value of $D$ is the largest one. Comprehensively considering the fusion results of the two tables, it can be judged that temperature, humidity is appropriate in the greenhouse now, but light intensity is insufficient. So it is necessary to do light supplement by using dysprosium lamp. Compared to high voltage sodium lamp and halide lamp, dysprosium lamp can make the light increased from $1000 l x$ to $4000 l x$. Because of this, dysprosium lamp can be used in raining days, fog days and evening. 


\section{Conclusions}

At present, Multisensor data fusion technology has been successfully applied to the automatic control, the military and aviation navigation. It is not a single technology, but an interdisciplinary comprehensive theory and method. We should actively learn the research results home and abroad. The research in the agricultural engineering area should be vigorously promoted. This paper through two-step data fusion, the fusion results will be sent into the knowledge base of expert system as the basis of inference in the expert system and then adds the experiences of agricultural expert, more accurate decision results can be got.

The combination of data fusion technology and agriculture expert system is the development direction of precision agriculture. With the continuous improvement and development of the data fusion technology, it is believed that technical support will be offered to informationization and better educated the agricultural production in the near future. The agricultural production will become more and more automated and precision. The pace of development of modern agricultural production in China will be speedup.

\section{References}

[1] Xu, F.: The Study of the Development of Establishment Agriculture in China, Shandong Agriculture University, pp. 1-5. (2008)

[2] (US) Hall (D.L.). Multisensor Data Fusion Manual, pp. 256-260. Publishing House of Electronics Industry, Pekin (2008)

[3] Kang, Y.: Data Fusion Theory and Application, pp. 165-168. Publishing house of Xi-Dian University, Sian (2006)

[4] Zhao, Z.: The Countermeasures of Developing Intellective Agriculture Expert System. China Science and Technology Review, 16-18 (2009)

[5] Liang, J.: The Research and Design of Intelligent Greenhouse Environment Temperature and Humidity Measurement and Control System, pp. 25-30. Taiyuan University of Technology (2005)

[6] Ding, W.: The Analysis of Greenhouse Environment Control and Situation of Greenhouse Simulation Modeled To The Current. Chinese Society of Agricultural Machinery. Journal of Agricultural Machinery 40(5), 44-48 (2009)

[7] Cheng, M., Yuan, H., et al.: The Research Based on Multisensor Data Fusion and Greenhouse Environment Control. Agricultural Mechanization Research 31(7), 213-217 (2009) 